\title{
On the Security of a Practical Identification Scheme
}

\author{
Victor Shoup \\ Bellcore, 445 South St., Morristown, NJ 07960 \\ shoup@bellcore.com
}

\begin{abstract}
We analyze the security of an interactive identification scheme. The scheme is the obvious extension of the original square root scheme of Goldwasser, Micali and Rackoff to $2^{m}$ th roots. This scheme is quitc practical, especially in terms of storage and communication complexity. Although this scheme is certainly not new, its security was apparently not fully understood. We prove that this scheme is secure if factoring integers is hard, even against active attacks where the adversary is first allowed to pose as a verifier before attempting impersonation.
\end{abstract}

\section{Introduction}

An identification scheme is an interactive protocol in which one party, $P$ (the prover), tries to convince another party, $V$ (the verifier), of its identity. The prover $P$ has a secret key that allows it, and no one else, to convince the verifier of its identity.

There are several types of attacks that an adversary, trying to impersonate $P$, may attempt, which we categorize as follows. In a passive attack, before the adversary tries to impersonate $P$, he tries to learn something about $P$ 's secret key by interacting with $P$; however, the adversary is not allowed to deviate from $V$ 's protocol (this attack includes eavesdropping). Clearly, security against only passive attacks is not very satisfactory, as $P$ must trust $V$ to follow the protocol.

In an active altack, the adversary interacts with $P$, perhaps several times, posing as $V$, but not necessarily following $V$ 's protocol. Security against active attacks is obviously preferable to security against only passive attacks.

There are stronger types of attacks that one may consider, such as those discussed in the paper of Bellare and Rogaway [1]. However, security against active attacks is sufficient in most practical situations where identification is the only goal, such as when a smart card proves its identity to an untrusted verification device in order to gain acces to some resource.

In this paper, we analyze the security of an identification scheme. The scheme is the obvious extension of the original square root scheme of Goldwasser, Micali and Rackoff [4] to $2^{m}$ th roots. This scheme is quite practical, especially in terms of storage and communication complexity. Although this scheme is certainly not new, its security was apparently not fully understood. We prove that-like the square root scheme-this scheme is secure against active attacks if factoring integers is hard. 


\section{The square root and $\mathbf{2}^{m}$ th root schemes}

Let us first recall the square root scheme. For a given security parameter $k$, a secret/public key pair is generated as follows. A modulus $n$ is constructed by multiplying two distinct, randomly selected primes, both of binary length $k$; also, an element $a \in \mathbf{Z}_{n}^{*}$ is chosen at random, and we set $b=a^{2}$. The public key is $(b, n)$, and the secret key is $a$.

Let $m$ be a parameter such that $2^{m}$ grows faster than any polynomial in $k$. The protocol then repeats the following $m$ times:

1. $P$ chooses $r \in Z_{n}^{*}$ at random, computes $x=r^{2}$, and sends $x$ to $V$.

2. $V$ chooses $e \in\{0,1\}$ at random, and sends $e$ to $P$.

3. $P$ computes $y=r \cdot a^{e}$ and sends $y$ to $V ; V$ accepts if $y^{2}=x \cdot b^{e}$, and rejects otherwise.

Upon termination, $V$ accepts if it accepted at every iteration, and otherwise rejects.

The results of Goldwasser, Micali, and Rackoff imply that the square root scheme is secure against active attacks if factoring is hard.

We consider the following variant, which might be called the $2^{m}$ th root scheme. For security parameter $k$, a secret/public key pair is generated as follows. A modulus $n$ is constructed by multiplying two distinct, randomly selected primes, both of binary length $k$, and both congruent to $3 \bmod 4$; also, an element $a \in \mathbf{Z}_{n}^{*}$ is chosen at random, and we set $b=a^{q}$, where $q=2^{m}$, and $m$ is a parameter such that $2^{m}$ grows faster than any polynomial in $k$. The public key is $(b, n)$, and the sccret key is $a$.

The protocol then executes the following-just once:

1. $P$ chooses $r \in \mathrm{Z}_{n}^{*}$ at random, computes $x=r^{q}$, and sends $x$ to $V$.

2. $V$ chooses $e \in\{0, \ldots, q-1\}$ at random, and sends $e$ to $P$.

3. $P$ computes $y=r \cdot a^{e}$ and sends $y$ to $V ; V$ accepts if $y^{q}=x \cdot b^{e}$, and rejects otherwise.

Clearly, the $2^{m}$ th root is scheme fairly cfficient, and is vastly superior to the square root scheme in terms of communication complexity.

Our proof of security does not show that this protocol is zero-knowledge (in the sense of [4]). Moreover, this scheme is not a proof of knowledge (in the sense of [2]). Indeed, observe that a prover $P^{\prime}$ that somehow knows $a^{2}$ can make $V$ accept with probability $1 / 2$, since $P^{\prime}$ can respond correctly to all even challenges $e$; however, given $a^{2}$ and $a^{2^{m}}$, we cannot efficiently compute $c$ such that $c^{2^{m}}=a^{2^{m}}$ (unless factoring is easy). As we shall see, this apparent lack of "soundness" is not really a problem at all, and is in fact crucial to our proof of security, which utilizes what might be called a partial zero-knowledge simulation technique.

\section{Other identification schemes}

There are many identification schemes in the literature; we mention just a few. 
There are several other variants of the square root scheme, but the only other one in the literature that is secure against active attacks if factoring is hard is the Fiat/Shamir (FS) scheme [3]. While the time complexities of the FS and the $2^{m}$ th root schemes are similar, the $2^{m}$ th root scheme has much smaller space and communication complexities.

The Guillou/Quisquarter (GQ) scheme $[6,7]$ is the same as the $2^{m}$ th root scheme, except that $2^{m}$ is replaced by an $m$-bit prime number. It is only known to be secure against passive attacks, provided that RSA-inversion is hard (a possibly stronger assumption than the hardness of factoring). It is mentioned in [6] that using a power of two would be possible, but no indication of the security of such a scheme is given.

Ong and Schnorr [10] propose a scheme that generalizes the FS and $2^{m}$ th root schemes, but only analyze its security against passive attacks. Ohta and Okamoto [8] discuss other generalizations of the FS scheme.

Other identification schemes are based on the hardness of the discrete logarithm problem. The scheme of Schnorr [11] is only known to be secure against passive attacks, provided the discrete logarithm problem in (a subgroup of) $\mathbf{Z}_{p}^{*}$, where $p$ is prime, is hard. The communication, space, and time complexities for the $2^{m}$ th root scheme and the Schnorr schemes are similar; however, the "on-line" time complexity for the prover in the Schnorr scheme is significantly smaller.

Okamoto [9] gives modifications of the GQ and Schnorr schemes which are proved secure, even against active attacks, under the same intractability assumptions of the corresponding original schemes. ${ }^{1}$ These schemes are slightly less efficient than the corresponding original schemes.

\section{Overview}

The rest of the paper is organized as follows: in $\S 2$, we formally state our definition of security; in $\$ 3$, we give a proof of security for the $2^{m}$ th root scheme; in $\S 4$, we prove that the generalization proposed by Ong and Schnorr is also secure against active attacks; we make some concluding remarks in $\S 5$.

\section{Definition of Security}

For conciseness and clarity, we adopt the notation of [5] for expressing the probability of various events. If $S$ is a probability space, then [S] denotes the set of elements in this space that occur with nonzero probability. For probability spaces $S_{1}, S_{2}, \ldots$, the notation

$$
\operatorname{Pr}\left[p\left(x_{1}, x_{2}, \ldots\right) \mid x_{1} \leftarrow S_{1} ; x_{2} \leftarrow S_{2} ; \ldots\right]
$$

denotes the probability that the relation $p\left(x_{1}, x_{2}, \ldots\right)$ holds when each $x_{i}$ is chosen, in the given order, from the corresponding probability space $S_{i}$.

1 That paper also suggests a scheme (Scheme 3) whose security is supposedly based on factoring, but the claims of security made in that paper are evidently false. 
A probabilistic algorithm $A$ on a specific input $x$ produces an output string according to some probability distribution. We denote by $A(x)$ the probability space of output strings.

Generally, an identification scheme $(G, P, V)$ consists of a probabilistic, polynomial-time algorithm $G$, and two probabilistic, polynomial-time, interactive algorithms $P$ and $V$ with the following properties.

1. 'I'he algorithm $G$ is a key-generation algorithm. It takes as input a string of the form $1^{k}$ (i.e., $k$ written in unary), and outputs a pair of strings $(S, I)$. $k$ is called the security parameter, $S$ is called a secret key, and $I$ is called a public key.

2. $P$ receives as input the pair $(S, I)$ and $V$ receives as input $I$. After an interactive execution of $P$ and $V, V$ outputs a 1 (indicating "accept") or a 0 (indicating "reject"). For a given $S$ and $I$, the output of $V$ at the end of this interaction is of course a probability space and is denoted by $(P(S, I), V(I))$.

3. A legitimate prover should always be able to succeed in making the verifier accept. Formally, for all $k$ and for all $(S, I) \in\left[G\left(1^{k}\right)\right],(P(S, I), V(I))=1$ with probability 1.

An adversary $\left(P^{\prime}, V^{\prime}\right)$ is a pair of probabilistic, polynomial-time, interactive algorithms. For a given secret/public key pair $(S, I)$, we denote by $\left(P(S, I), V^{\prime}(I)\right)$ the string $h$ output by $V^{\prime}$ (on input $I$ ) after interacting with $P$ (on input $(S, I)$ ) some number of times. Note that these interactions are performed sequentially. Again, for a given $S$ and $I,\left(P(S, I), V^{\prime}(I)\right)$ is a probability space. The string $h$ (a "help string") is used as input to $P^{\prime}$, which attempts to make $V$ (on input $I$ ) accept.

Definition 1. An identification scheme $(G, P, V)$ is secure against active attacks if for all adversaries $\left(P^{\prime}, V^{\prime}\right)$, for all constants $c>0$, and for all sufficiently large $k$,

$$
\operatorname{Pr}\left[s=1 \mid(S, I) \leftarrow G\left(1^{k}\right) ; h \leftarrow\left(P(S, I), V^{\prime}(I)\right) ; s \leftarrow\left(P^{\prime}(h), V(I)\right)\right]<k^{-c} .
$$

\section{Security of the $\mathbf{2}^{m}$ th root scheme}

Our proof of security is based on the assumption that factoring is hard. We make this assumption precise.

For $k \geq 5$, let $H_{k}$ be the probability space consisting the uniform distribution over all integers $n$ of the form $n=p_{1} \cdot p_{2}$, where $p_{1}$ and $p_{2}$ are distinct primes of binary length $k$, and $p_{1} \equiv p_{2} \equiv 3(\bmod 4)$.

The Factoring Intractability Assumption (FIA) is the following assertion:

for all probabilistic, polynomial-time algorithms $A$, for all $c>0$, and for all sufficiently large $k$,

$\operatorname{Pr}\left[x\right.$ is a nontrivial factor of $\left.n \mid n \leftarrow H_{k} ; x \leftarrow A(n)\right]<k^{-c}$. 
We shall prove:

Theorem 1. Under the $F I A$, the $2^{m}$ th root scheme is secure against active attacks.

To prove this theorem, we show that any adversary that succeeds in an impersonation attempt with non-negligible probability can be converted into a probabilistic, polynomial-time factoring algorithm that succeeds with nonnegligible probability. This is Lemma 1 below.

Let $\left(P^{\prime}, V^{\prime}\right)$ be such an adversary. Then there are polynomials $T_{i}(k), N_{i}(k)$, $T_{o l}(k)$, and $T_{p}(k)$ as follows.

$T_{i}(k)$ is a bound on the time required for $V^{\prime}$ to run the protocol once with $P$, and includes the computation time of $P$. This computation is done "on-line," and thus will typically have to be fast.

$N_{i}(k)$ is a bound on the number of times $V^{\prime}$ runs the protocol with $P$. This will typically be small.

$-T_{o l}(k)$ is a bound on the "off-line" time for $V^{\prime}$; i.e., all time spent by $V^{\prime}$ other than running the protocol with $P$.

- $T_{p}(k)$ is a bound on the running-time of $P^{\prime}$ and $V$. This computation is also "on-line," and thus will also typically have to be fast.

For a given public key $(b, n)$ and "help string" $h$, let

$$
\epsilon(h, b, n)=\operatorname{Pr}\left[\left(P^{\prime}(h), V(b, n)\right)=1\right] .
$$

Then there exist polynomials $Q_{1}(k)$ and $Q_{2}(k)$ and an infinite set $K \subset Z_{>0}$ such that for all $k \in K$, the probability

$$
\operatorname{Pr}\left[\epsilon(h, b, n) \geq Q_{2}(k)^{-1} \mid(a,(b, n))-G\left(1^{k}\right) ; h \leftarrow\left(P(a,(b, n)), V^{\prime}(b, n)\right)\right]
$$

is at least $Q_{1}(k)^{-1}$.

Lemma 1. Assume an adversary as above. Then there is a probabilistic factoring algorithm $A$ that runs in time

$$
O\left(\left(N_{i}(k) T_{i}(k)+T_{p}(k)\right) Q_{2}(k)+T_{o l}(k)\right)
$$

such that for all sufficiently large $k \in K$,

$\operatorname{Pr}\left[x\right.$ is a nontrivial factor of $\left.n \mid n \leftarrow H_{k} ; x \leftarrow A(n)\right] \geq Q_{1}(k)^{-1 / 8}$.

The special form of integers $n \in\left[H_{k}\right]$ implies that the operation of squaring on $\mathbf{Z}_{n}^{*}$ acts as a permutation on the subgroup $\left(Z_{n}^{*}\right)^{2}$ of squares. Also, every square $z$ in $\mathbf{Z}_{n}^{*}$ has precisely 4 square roots, exactly one of which is also a square. For convenience, and quite arbitrarily, for $w \in \mathbf{Z}_{n}^{*}$ we define $C(w) \in\{1, \ldots, 4\}$ to be the relative position of $w$ among the 4 square roots of $w^{2}$ when viewed as integers between 0 and $n-1$. 
We now describe and at the same time analyze our factoring algorithm. In all statements concerning probabilities, the underlying probability space consists of the random choice of the input $n$ and the coin tosses of the algorithm.

On input $n \in\left[H_{k}\right]$, the algorithm begins by computing $l$ as the smallest nonnegative integer with $2^{l} \geq Q_{2}(k)$. We require that $0 \leq l \leq m-1$, which will hold for all sufficiently large $k$, since we are assuming that $2^{m}$ grows faster than any polynomial in $k$.

The algorithm runs in three stages, as follows.

Stage 1. This stage takes as input $n$, runs in time $O\left(N_{i}(k) T_{i}(k) Q_{2}(k)+T_{o l}(k)\right)$, and outputs $(v, b, h)$ where $v, b \in \mathbf{Z}_{72}^{*}$ with $v^{2^{m-1}}=b$ and $h$ is a "help string." Moreover, we have:

(i) if $k \in K$, then $\operatorname{Pr}\left[\epsilon(h, b, n) \geq Q_{2}(k)^{-1}\right] \geq Q_{1}(k)^{-1} / 2$;

(ii) the distribution of $C(v)$ is uniform and independent of that of $(h, b, n)$.

This stage runs as follows. We choose $v \in \mathbf{Z}_{n}^{*}$ at random and compute $b=$ $v^{2^{m-1}}$. Note that the distribution of $(b, n)$ is independent of $C(v)$, and is the same as that of an ordinary public key - this is because squaring permutes $\left(\mathbf{Z}_{n}^{*}\right)^{2}$, and so $b$ should be, and is, just a random square. We then simulate the interaction $\left(P(\cdot, b, n), V^{\prime}(b, n)\right)$. This is complicated by the fact that we do not have at hand a value $a$ such that $a^{2^{m}}=b$, but rather $v$ with $v^{2^{m-1}}=b$.

We employ a variation of a zero-knowledge simulation technique introduced by Goldwasser, Micali, and Rackoff [4], which might be called partial zeroknowledge simulation. We replace the identification protocol with the following:

$1^{\prime} . P$ chooses $e_{0}^{\prime} \in\left\{0, \ldots, 2^{l}-1\right\}$ at random, chooses $r \in \mathbf{Z}_{n}^{*}$ at random, computes $x=r^{2^{m}} \cdot b^{-e_{0}^{\prime}}$, and sends $x$ to $V^{\prime}$.

$2^{\prime}$. $V^{\prime}$ computes a challenge $e \in\left\{0, \ldots, 2^{m}-1\right\}$ and sends $e$ to $P$.

$3^{\prime}$. $P$ writes $e=\epsilon_{1} 2^{l}+e_{0}$. If $e_{0} \neq e_{0}^{\prime}$, we go back to step $1^{\prime}$ ("undoing" the computation of $V^{\prime}$ ). Otherwise, $P$ computes $y=r \cdot v^{e_{1}}$ and sends $y$ to $V^{\prime}$.

An easy calculation shows that if $e_{0}=e_{0}^{\prime}$, then $y^{2^{m}}=x \cdot b^{e}$; moreover, it is easily seen that the distribution of $(x, y)$ is precisely the same as it would be in the original protocol, and is independent of $C(v)$.

The expected number of loop iterations until $e_{0}=e_{0}^{\prime}$ is $2^{l}$. Over the course of the entire interaction between $P$ and $V^{\prime}$, the expected total number of challenges is at most $2^{l} N_{i}(k)$. If the total number of challenges ever exceeds twice this amount, we quit and output an arbitrary $h$; otherwise, we output the $h$ that $V^{\prime}$ outputs. We also output $v$ and $b$.

That completes the description of Stage 1. All of the claims made above about this stage are easily verified.

Stage 2. This stage takes as input $h, b$, and $n$ from above, and runs in time $O\left(T_{p}(k) Q_{2}(k)\right)$. It reports failure or success, and upon success outpuls $z \in \mathbf{Z}_{n}^{*}$ and $f \in\left\{0, \ldots, 2^{m}-1\right\}$ such that $z^{2^{m}}=b^{f}$ and $f \neq 0\left(\bmod 2^{l+1}\right)$. The probability of success, given that $\epsilon(h, b, n) \geq Q_{2}(k)^{-1}$, is at least $1 / 2$. 
Let $\epsilon=\epsilon(h, b, n)$, and assume $\epsilon \geq Q_{2}(k)^{-1}$.

We use a slight variation of an argument in Feige, Fiat, and Shamir [2]. Consider the Boolean matrix $M$ whose rows are indexed by the coin toss string $\omega$ of $P^{\prime}$ and whose columns are indexed by the challenges $e$ of $V . M(\omega, e)=1$ if and only if this choice of $\omega$ and $e$ causes $V$ to accept.

Call a row $\omega$ in $M$ heavy if the fraction of l's in the row is at least $3 \epsilon / 4$. Observe that the fraction of 1 's in the matrix that lie in heavy rows is at least $1 / 4$. Now consider a heavy row $\omega$, and a challenge $e$ such that $M(\omega, e)=1$. Consider the fraction of challenges $e^{\prime}$ with the property that $M\left(\omega, \epsilon^{\prime}\right)=1$ and $e^{\prime} \not \equiv e\left(\bmod 2^{i+1}\right)$. This fraction is at least

$$
3 \epsilon / 4-2^{-3-1} \geq 2^{-l-2}>Q_{2}(k)^{-1} / 8 .
$$

Stage 2 runs as follows. Run $\left(P^{\prime}(h), V(b, n)\right)$ up to $O\left(Q_{2}(k)\right)$ times, or until $V$ accepts. If $V$ accepts, we have a relation $y^{2^{m}}=x b^{e}$. Fixing the coin tosses of $P^{\prime}$, run the interaction again up to $O\left(Q_{2}(k)\right)$ times, or until $V$ accepts again with a challenge $e^{\prime} \not \equiv e\left(\bmod 2^{l+1}\right)$. If $V$ accepts with such a challenge, then we have another relation $\left(y^{\prime}\right)^{2^{m}}=x b^{e^{\prime}}$.

The above can be repeated some constant number of times to raise the probability of finding two such relations to at least $1 / 2$. Upon finding two such relations, and ordering them so that $e>e^{\prime}$, we output $z=y / y^{\prime}$ and $f=e-e^{\prime}$.

That completes the description and analysis of Stage 2.

Stage 3 of our factoring algorithm is executed only if Stage 2 succeeds.

Stage 3. This stage takes as input $n$, the value $v$ from Stage 1 , and the values $z$ and $f$ from Stage 2. It runs in time $O\left(k^{3}\right)$. The probability that it outputs a nontrivial factor of $n$, given that Stage 2 succeeded, is $1 / 2$.

This stage runs as follows. We write $f=u 2^{t}$, where $u$ is odd and $0 \leq t \leq l$. Set $w=z^{2^{l-t}}$. We claim that with probability $1 / 2, \operatorname{gcd}\left(v^{u}-w, n\right)$ is a nontrivial factor of $n$.

To see this, first note that $w^{2^{m-l+t}}=b^{u 2^{t}}$, and hence $w^{2^{m-l}}=b^{u}$ (as squaring permutes $\left(\mathbf{Z}_{n}^{*}\right)^{2}$ ). We have $\left(v^{u}\right)^{2^{m-l}}=b^{u}$ and hence $\left(v^{u}\right)^{2}=w^{2}$ (again, squaring permutes $\left.\left(\mathbf{Z}_{n}^{*}\right)^{2}\right)$. But since $u$ is odd, and by the independence of $C(v)$ and $w, C\left(v^{u}\right)$ has a uniform distribution independent from $C(w)$, and so with probability $1 / 2, v^{u} \neq \pm w$. In this case, $\operatorname{gcd}\left(v^{u}-w, n\right)$ is a nontrivial factor of $n$.

That completes the description and analysis of Stage 3.

It follows that for sufficiently large $k \in K$, the overall success probability of our factoring algorithm is at least

$$
Q_{1}(k)^{-1 / 2} \times 1 / 2 \times 1 / 2=Q_{1}(k)^{-1} / 8 .
$$

That completes the description and analysis of our factoring algorithm, and the proof of Lemma 1. 


\section{The Ong and Schnorr Generalization}

In this section, we consider a generalization of the $2^{m}$ th root scheme that was proposed by Ong and Schnorr [10], and prove that it is secure against active attacks if factoring is hard.

In this scheme, the modulus $n$ is chosen precisely as before. There are two parameters $s$ and $m$, chosen so that $2^{s m}$ grows faster than any polynomial in $k$. Set $q=2^{m}$. A private key consists of a list $a_{1}, \ldots, a_{s}$ of randomly chosen elements of $\mathbf{Z}_{n}^{*}$; the corresponding public key consists of $b_{1}, \ldots, b_{s} \in \mathbf{Z}_{n}^{*}$, where $b_{i}=a_{i}^{q}$ for $1 \leq i \leq s$.

The protocol then runs as follows:

1. $P$ chooses $r \in \mathrm{Z}_{n}^{*}$ at random, computes $x=r^{q}$, and sends $x$ to $V$.

2. $V$ chooses $e_{1}, \ldots, e_{s} \in\{0, \ldots, q-1\}$ at random, and sends $e_{1}, \ldots, e_{s}$ to $P$.

3. $P$ computes $y=r a_{1}^{e_{1}} \cdots a_{s}^{e_{s}}$ and sends $y$ to $V ; V$ accepts if $y^{q}=x b_{1}^{e_{1}} \cdots b_{s}^{e_{s}}$, and otherwise rejects.

By setting $s=1$, this scheme degenerates to the $2^{m}$ th root scheme. This scheme allows one to somewhat reduce the time complexity at the expense of increasing the key size.

Theorem 2. Under the FIA, the general Ong and Schnorr scheme is secure against active attacks.

The proof is similar to that of Theorem 1; we sketch the differences. In the factoring algorithm, the value $l$ is chosen to be the least nonnegative integer such that $2^{s(l+1)} \geq 2 Q_{2}(k)$. As before, we require that $0 \leq l \leq m-1$, but this will hold for all sufficiently large $k$.

In Stage 1 , for $1 \leq i \leq s$, we choose $v_{i} \in \mathbf{Z}_{n}^{*}$ at random, and compute $b_{i}=v_{i}^{2^{m-l}}$. Then we perform the same simulation as in Stage 1, except this time, we have to guess the low-order $l$ bits of each of the $s$ challenges.

Stage 2 is casily modified so as to obtain $z \in \mathbf{Z}_{n}^{*}$ and integers $f_{1}, \ldots, f_{s}$ such that $z^{2^{m}}=\prod_{i} b_{i}^{f_{i}}$ and not all $f_{i}$ are divisible by $2^{i+1}$.

In Stage 3 , for $1 \leq i \leq s$, write $f_{i}=u_{i} 2^{t_{i}}$, and let $t=\min \left\{t_{i}\right\} \leq l$. Then it is easy to see that with probability $1 / 2$,

$$
\operatorname{gcd}\left(z^{2^{1-t}}-\prod_{i=1}^{s} v_{i}^{u_{i} 2^{t_{i}-t}}, n\right)
$$

is a nontrivial factor of $n$.

\section{Conclusion}

\subsection{Constructiveness and efficiency of the reduction}

Although our proof of security is valid, it is open to a couple of criticisms. First, it is not entirely constructive, since to build our factoring algorithm, we need not 
only descriptions of the adversary's algorithms, but also the polynomials $Q_{2}(k)$ and $N_{i}(k)$. By allowing our factoring algorithm to have just an expected running time bound, we could do without $N_{i}(k)$. However, it is not clear if we can do without $Q_{2}(k)$. Second, in comparison, say, with the reduction from factoring to impersonating one obtains with the FS scheme, ours is less efficient, since our factoring algorithm has to repeat computations of $V^{\prime}$ many times. Compensating for this is the fact that the computations that need to be repeated are all "online," and so presumably fast.

\subsection{Multi-user environment}

We have stated the protocols and definition of security from the point of view a single user. Consider a a system consisting of many (but polynomial in $k$ ) users. Before attempting an impersonation, we allow an adversary to interact arbitrarily with all users in the system in an arbitrary fashion, interleaving communications arbitrarily. We also allow an adversary to corrupt any users it wants, obtaining their private keys upon demand. After this interaction, the adversary tries to impersonate a non-corrupted user of its choice. Note that the adversary makes this choice dynamically; if the choice were static, the analysis of the multi-user case would trivially reducc to the single-user case.

To reduce factoring to impersonating in this case, we can use the obvious technique of first picking a user at random and giving them the number we want to factor, and generating ordinary key pairs for all other users. We then hope that the adversary picks the user with our number.

As with the FS scheme, all users can share the same modulus $n$ in the $2^{m}$ th root scheme. However, to reduce factoring to impersonating in this case, we still have to pick a user at random, and give this uscr $v, b \in \mathbf{Z}_{n}^{*}$ with $v^{2^{m-l}}=b$. Again, the other users get ordinary key pairs, and we have to hope that the adversary chooses to impersonate our user. This is unlike the FS scheme, where every user gets ordinary key pairs, and the impersonation of any user factors $n$ with high probability.

\section{$5.3 \quad$ Using general moduli}

Our proofs of Theorems 1 and 2 relied on the fact that the prime factors of $n$ were both congruent to 3 mod 4 . However, this is not a serious restriction, and it is not hard to prove that these theorems hold for random primes. It is not clear if this is of any theoretical or practical value, and from a security perspective, it seems advisable to stick with the special moduli.

\section{References}

1. M. Bellare, J. Kilian, and P. Rogaway. Entity authentication and key distribution. In Advances in Cryptology-Crypto '93, pages 232-233, 1993. 
2. U. Feige, A. Fiat, and A. Shamir. Zero-knowledge proofs of identity. J. Cryptology, 1:77 $94,1988$.

3. A. Fiat and A. Shamir. How to prove yourself: practical solutions to identification and signature problems. In Advances in Cryptology-Cryplo '86, pages 186-194, 1986.

4. S. Goldwasser, S. Micali, and C. Rackoff. The knowledge complexity of interactive proof systems. SIAM J. Comput., 18:186-208, 1989.

5. S. Goldwasser, S. Micali, and R. Rivest. A digital signature scheme secure against adaptive chosen-message attacks. SIAM J. Comput., 17:281-308, 1988.

6. L. Guillou and J. Quisquater. A "paradoxical" identity-based signature scheme. resulting from zero-knowledge. In Advances in Cryptology-Crypto '88, pages 216$231,1988$.

7. L. Guillou and J. Quisquater. A practical zero-knowledge protocol fitted to security microprocesors minimizing both transmission and memory. In Advances in Cryptology-Eurocrypt '88, pages 123-128, 1988.

8. K. Ohta and T. Okamoto. A modification of the Fiat-Shamir Scheme. In Advances in Cryptology-Crypto '88, pages 232-243, 1988.

9. T. Okamoto. Provably secure and practical identification schemes and corresponding signature schemes. In Advances in Cryptology-Crypto '92, pages 31-53, 1992.

10. H. Ong and C. Schnorr. Fast signature generation with a Fiat Shamir-like scheme. In Eurocrypt, pages 432-440, 1990.

11. C. Schnorr. Efficient signature generation by smart cards. J. Cryptology, 4:161$174,1991$. 